\title{
Lösungsmöglichkeiten nach fehlgeschlagenen Spondylodesen an der Halswirbelsäule
}

\author{
Peter Seykora, Anton Kathrein, Michael Blauth
}

\section{Zusammenfassung}

Wenig operative Verfahren an der Wirbelsäule haben über die letzten Jahrzehnte eine ähnliche Standardisierung wie ventrale, dorsale oder kombinierte Stabilisierungsoperationen an der Halswirbelsäule erfahren. Der Grund liegt einerseits in den klaren zugrunde liegenden biomechanischen Prinzipien, den anatomischen Verhältnissen, welche wenig $\mathrm{Zu}-$ gangsspielraum bieten, und andererseits in der bis dato großen Anzahl weltweit erfolgreich durchgeführter und jahrelang nachuntersuchbarer Eingriffe. Weiterentwicklungen und Innovation müssen sich, was Fehlschläge und Komplikationen und nicht zuletzt auch die Kosten angeht an diesen Standards messen lassen. Misserfolge liegen selten, wie dargestellt systemimmanent, also der Methode oder dem Implantat zuschreibbar, sondern sie sind oft das Endresultat von präoperativen Fehlentscheidungen, Fehleinschätzungen des klinischen und radiologischen $\mathrm{Zu}-$ standsbildes, Missachtung biologischer und biomechanischer Grundsätze, Überschätzung implantatme- chanischer Möglichkeiten und postoperativ mangelnden Einsicht in offensichtliche klinische oder radiologische Fehlentwicklungen. Ebenso erscheint ein blindes Vertrauen in biomechanischen Untersuchungen, welche beispielsweise dorsale oder kombinierte Verfahren aufgrund errechneter höherer Stabilität forcieren, nicht opportun. Diese präparatgebunden „nekromechanischen“ Untersuchungen müssen die klinischen Ergebnisse eben außer Acht lassen und können so nur einen Beitrag zur Gesamtproblematik liefern. Neuerungen und Fortschritte in operativer Technik oder Implanatdesign sind zweifellos wichtig, sie dürfen aber nicht den Blick auf hinlänglich beschriebene Komplikationen trüben, um in weiterer Folge bekannte aber eben vergessene Fehler wieder neu zu machen. Sie geben vielmehr dem Operateur im Rahmen differenzierter Eingriffe an der Halswirbelsäule, insbesonders im Revisonsfall exzellente Möglichkeiten in die Hand bei geringer neuerlicher Zugangsmorbidität und Schonung angrenzender Segmente, übungsstabile Verhältnisse zu schaffen.
Grundlage dieses Beitrages ist nicht die Wertung und Gewichtung der Literatur, sondern vielmehr die Darstellung bekannter intra- sowie postoperativer Komplikationen nach Fusionsoperationen an der Halswirbelsäule einschließlich möglicher Lösungsansätze.

\section{Zugang zur Halswirbelsäule}

Bis vor geraumer Zeit war die Zugangsfrage zur Halswirbelsäule eine vom Verursacherprinzip geprägte Diskussion, dahingehend, dass der Ort der Läsion die Frage des dorsalen oder ventralen Vorgehens entscheidend beeinflusste [4].

Anatomisch und im Hinblick auf die $\mathrm{Zu}-$ gangsmorbidität wurde der anteromediane Zugang favorisiert, bei welchem nur anatomische Spatien eröffnet und durchquert werden müssen, wohingegen das dorsale Vorgehen eine mehr oder weniger rigorose Abpräparation der autochtonen Muskulatur notwendig macht [5].

Seit mehreren Jahren setzt sich eine differenziertere Anschauungsweise durch, welche einerseits von anatomischen Überlegungen, andererseits auch von zunehmender Erfahrung geprägt ist. So zeigte sich beispielsweise, dass traumatische Läsionen im zervikalem Gelenksbereich, auch dorsal verhakte Zustände, durchaus rein von ventral angegangen und gelöst werden können [6].

bekannte Komplikationen nach alleiniger Spaninterposition an der Halswirbelsäule wie Nachsinterungen, Korrekturverluste und Spandislokationen deutlich reduziert werden.

Die ventrale interkorporelle Plattenspondylodese erfuhr in der Folge eine weltweite Verbreitung sowohl im Rahmen von traumatologischen als auch von degenerativen und tumorchirurgischen Anwendungen. Vielfach wurde über die Operationstechnik, die Zugangsproblematik, und auch - zumeist retrospektiv - über Ergebnisse im Schrifttum berichtet.
Auch bei Zervikalstenosen auf mehreren Etagen weist die Literatur eher in Richtung einer ventralen Dekompression und Fusion und weg von Laminektomie oder Lamionoplastie [7].

Primäres dorsoventrales Vorgehen ist heute eine Domäne der Tumorchirurgie sowie hochgradig instabiler Zustände wie zum Beispiel Frakturen bei M. Bechterew [8].

Sonderfälle stellen ein- oder beidseitig verhakte Luxationen dar. Während beid-
OP-JOURNAL 2004; 20: 182-187

(c) Georg Thieme Verlag KG Stuttgart · New York 
seitig verhakte Luxationen zumeist hoch instabil sind und leicht gedeckt reponiert und auch nur von ventral behandelt werden können, lassen sich einseitig verhakte Frakturen nicht selten schwer reponieren. In beiden Fällen muss vor jedem Eingreifen, sei es nun gedeckt oder offen, radiologisch abgeklärt werden, ob auf selber Höhe disloziertes freies Bandscheibenmaterial dorsal der Wirbelkörperhinterkante darstellbar ist, welches sich im Rahmen eines gedeckten Repositionsmanövers nach dorsal verlagern könnte. In diesem Fall würde ein mehrstufiges Vorgehen mit Diskusresektion von ventral, Enthakung und Spondylodese von dorsal und schließlich ventraler Fusion und Instrumentation angezeigt sein.

Die Entscheidung, ob von ventral oder dorsal zugegangen wird, richtet sich zudem auch nach der Physiognomie des Patienten. Beispielsweise kann eine ventrale Schraubenosteosynthese bei Densfraktur durch die Kombination „kurzer Hals" und „fassförmiger Thorax" unmöglich werden. In diesem Fall ist die dorsale Fusion der einzige Ausweg.

Im Rahmen von Revisionseingriffen stellt sich neben den oben genannten Punkten auch die Frage nach dem rechts- oder linksseitigem Zugang zur ventralen HWS. Generell besteht die Empfehlung, dass bei Eingriffen unterhalb des Niveaus C5/C6 dem linksseitigen Zugang der Vorzug zu geben ist, nachdem dort der N. laryngeus recurrens aufgrund anatomischer Gegebenheiten besser geschützt liegt.

Im Revisionsfall sollte man nicht automatisch den kontralateralen anteromedianen Zugang wählen, um - bei vernarbten Verhältnissen auf der ipsilateralen Seite dem erhöhten Risiko einer Rekurrenslähmung aus dem Wege zu gehen. Präoperativ ist vielmehr eine laryngoskopische Untersuchung angezeigt, um abzuklären, ob schon eine Parese vorliegt. In diesem Fall sollte dem ipsilateralem Zugang, eventuell mit intraoperativem Monitoring des N. recurrens, der Vorzug gegeben werden, um nicht im Komplikationsfall eine partielle oder totale Aphonie zu provozieren.

Bei allen ventralen Eingriffen an der HWS empfiehlt sich zudem die Einlage einer dünnen Magensonde, im Revisionsfall die Einlage eines dicken Magenschlauches. Beide sind bei der Präparation hilfreich und minimieren das Risiko einer iatrogenen Ösophagusperforation deut- lich. Nach abgeschlossener Präparation kann letzterer intraoperativ mühelos durch den Anästhesisten entfernt werden.

\section{Instrumentationsbedingte, intraoperative Komplikationen}

Dorsale und ventrale Eingriffe an der Halswirbelsäule sind in Technik und Vorgehen standardisierte Eingriffe. Trotzdem werden doch immer wieder ähnliche intraoperative Probleme rund um das Implantathandling berichtet.

\section{Ventrale Eingriffe}

Die ventrale Plattenspondylodese nach interkorporeller Spaninterposition folgt dem biomechanischen Prinzip der Neutralisation: Die auf den interkorporell eingebrachten Knochenspan einwirkende Rotations- oder Scherkräfte werden durch die ventrale Platte geleitet und so neutralisiert. Hier spielt es theoretisch keine wesentliche Rolle, ob das Implantat etwas seitlich versetzt oder leicht rotiert eingebracht wird, solange gewährleistet ist, dass die Schrauben in den zu fusionierenden Wirbelkörpern sicheren Halt finden.

Es empfiehlt sich also zu Implantaten zu greifen, welche einerseits nicht $\mathrm{zu}$ weit nach lateral ausladen, um genügend Spielraum zu haben. Weiterhin sollten nach einer Fehlbohrung oder im Rahmen eines Revisionseingriffes „Salvageschrauben“ mit einem dickeren Kerndurchmesser zur Verfügung stehen. Des Weiteren muss das Platten-Schraubendesign eine ausreichende Winkelvariabilität bieten (Abb.1).

Diese Implantateigenschaften bewähren sich einerseits bei der Teilkorporektomie im Traumafall, bei welcher nur ein versatiles und „schlankes“ Implantat die Schraubenverankerung im restlich verbliebenen Wirbelkörper noch ermöglicht, ansonsten eine bisegmentale Fusion nach Wirbelkörperresektion die eindeutig schlechtere Alternative darstellen würde.

Vor allem diese genannten Implantateigenschaften machen uns zu eindeutigen Befürwortern winkelinstabiler Platten-Schrauben-Konstrukte an der ventralen HWS. Zudem sahen wir aber vor allem im Rahmen eigener biomechanischer Untersuchungen keinen signifikanten Unterschied zwischen konventionellen $\mathrm{H}$ Platten und den ventralen Verriegelungsplatten $[9,10]$,
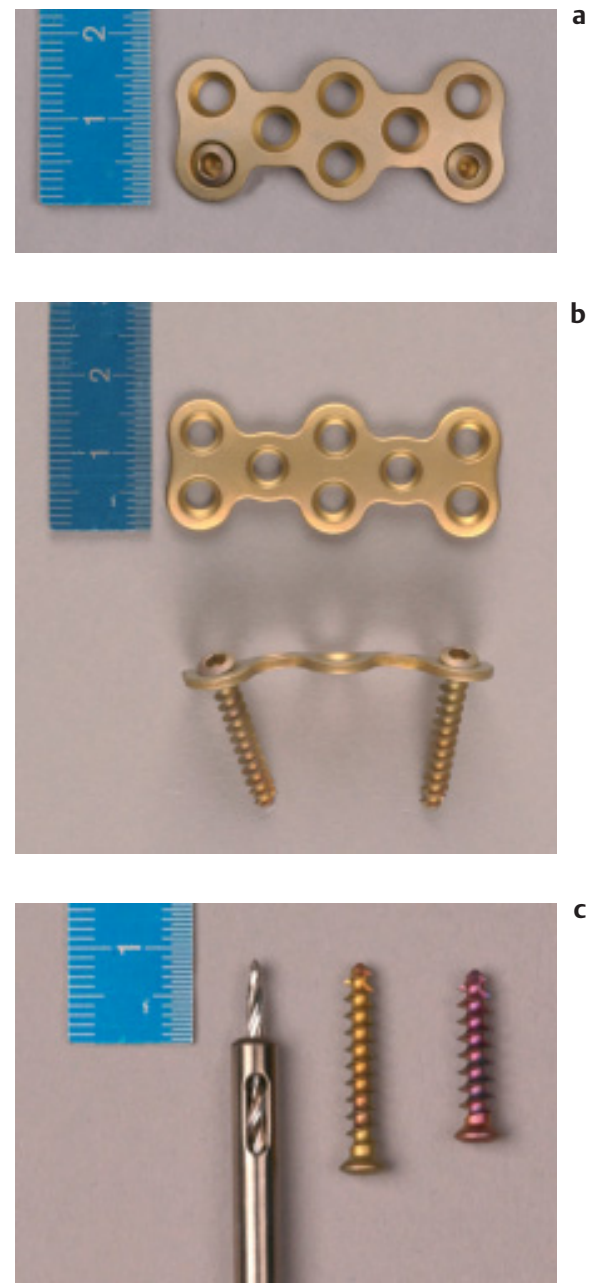

Abb.1 Das abgebildete Instrumentarium in Titanausführung zur ventralen Plattenspondylodese an der HWS (Stryker Leibinger GmbH, Freiburg) zeichnet sich durch einerseits geringe laterale Ausladung und andererseits schlanke Bauhöhe mit homogem glatten Übergang zwischen Schraubenkopf und Platte bei doch ausreichender Winkelvariabilität aus (a und b). Abb. 1 c zeigt den Bohrer mit fixem Tiefenanschlag von $10 \mathrm{~mm}$, sowie die selbstschneidenden Schrauben in Standardausführung (gold) und die "Salvageschraube" mit dickerem Kerndurchmesser (rot).

Im ventralen Revisionsfall verbleibt so oft noch genügend Spielraum, um das Implantat neu zu positionieren und eventuell unter Anwendung der genannten „Salvageschrauben“ zu fixieren.

Im seltenen Fall eines intraoperativen Liquorflusses aus einem Schraubenloch infolge iatrogener Duraperforation kann diese Salvageschraube in Kombination mit einer kleinen Fibrinplombe als Verschluss dienlich sein. Eine frühpostoperative neurologische Beurteilung inkl. post- 
operativen Myelographie ist jedoch dringend angezeigt.

\section{Dorsale Eingriffe}

Dorsale Spondylodesen folgen fast immer dem Prinzip der Zuggurtung. Eine Ausnahme stellt die früher zur Anwendung kommende dorsale Gelenkmassivplatte dar. Diese dem Abstützungsprinzip folgende Instrumentation fand früher bei Defektfrakturen eines oder beider Gelenkfacetten in Kombination mit einer dorsalen Cerclage oder Platte ihre Anwendung. Die Entwicklung dorsaler win- kelstabiler Fixateursysteme machte diese Anwendung zwischenzeitlich unnötig.

Aufgrund anatomischer Gegebenheiten sind den Schraubeneintrittspunkten an der dorsalen HWS enge Grenzen gesetzt. Im Revisionsfall kann daher nur schwer eine andere Schraubenposition nach neuerlichem Bohren gefunden werden. Die genannten Fixateursysteme bieten aufgrund ihrer Winkelvariabilität in der Stabschraubenverbindung diese Versatilität und besitzen in Form von Laminahaken weitere Möglichkeiten der dorsalen Implantatfixation. Im Falle einer nur einseitig ausreichenden Fixation stellt die
Anbringung eines Querstabilisators oft eine ausreichende Maßnahme zur zusätzlichen Versteifung des Konstruktes dar. Im Routinefall sollte aber auf dessen Anwendung im Hinblick auf eine optimalere Readaptation der Muskulatur an die dorsalen Wirbelstrukturen verzichtet werden.

Eine weitere Rettungsmöglichkeit bietet die zusätzliche Verwendung einer klassischen, um die Processus spinosi gelegte Drahtcerclage in Kombination mit interspinöser Spaninterposition. Dieses Vorgehen setzt natürlich intakte dorsale Wirbelstrukturen voraus.
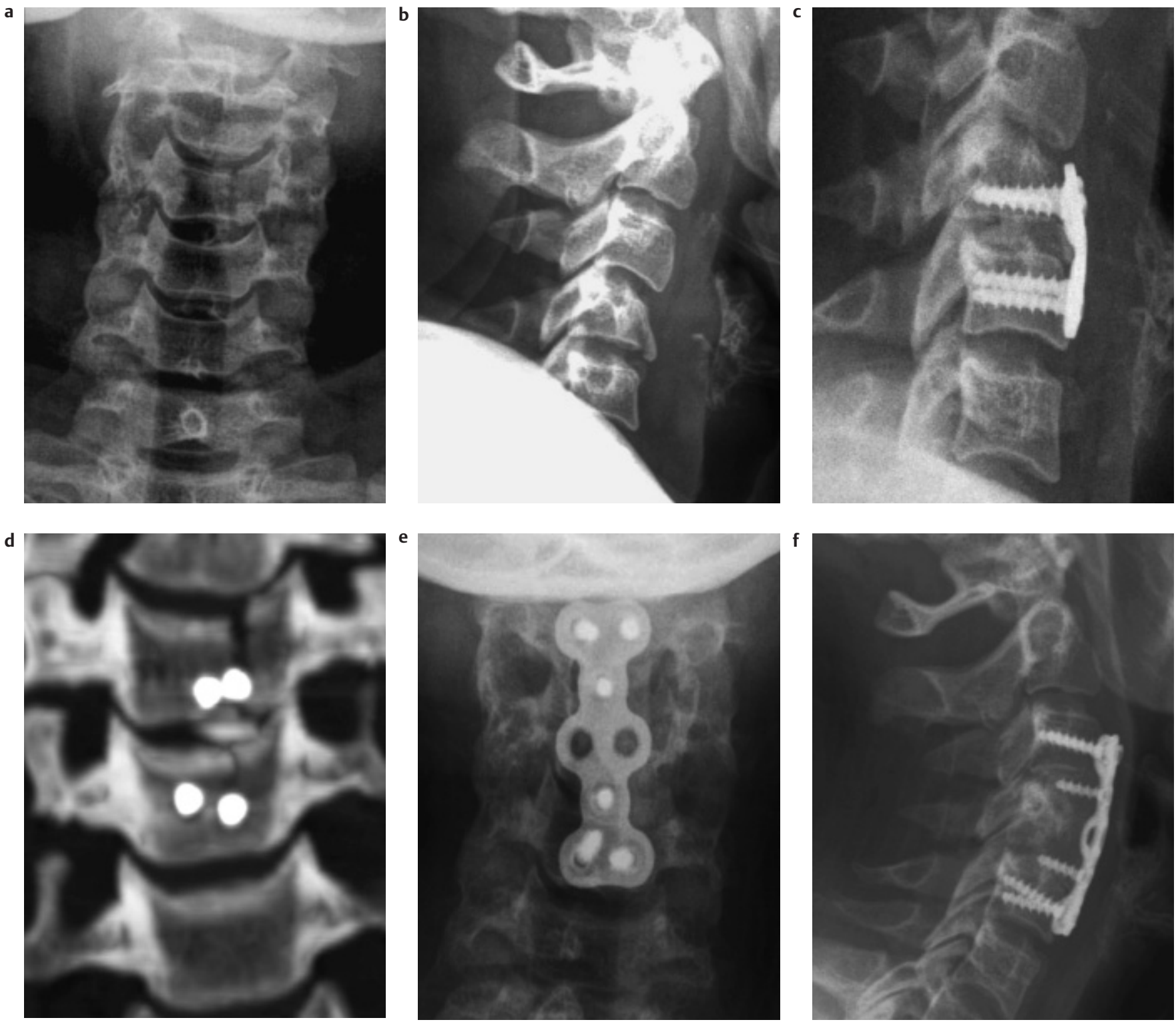

Abb. 2 S.B. 28 Jahre Pkw-Insassin: Berstungsspaltbruch C4 mit subtotalem Querschnitt (a und b). Am Aufnahmetag interkorporelle monosegmentale Fusion C4/C5. Wenige Tage später zunehmende Rekyphosierung und Instabilität im Segment C3/C4. Die CT-Kontrolle zeigt den
Berstungsspaltbruch C4, die Schrauben finden im Bruchspalt keinen ausreichenden Halt (c und d). Nach Teilkorporektomie C4 und bisegmentaler interkorporeller Fusion und Instrumentation C3 bis C5 (e und $\mathbf{f}$ ). 


\section{Frühkomplikationen nach Spondylodesen der HWS}

\section{Unzureichende Reposition}

Die Wiederherstellung achsengerechter Verhältnisse nach posttraumatischen Fehlstellungen gehört neben der Wiedererlangung der schmerzfreien Übungsstabilität zu den Grundanforderungen chirurgischen Handelns an der Wirbelsäule. Sowohl dorsale als auch ventrale Verfahren zielen darauf ab, die physiologische Krümmung der Halswirbelsäule wiederherzustellen und mittels geeigneter Maßnamen bis zur knöchernen Konsolidierung auch zu halten.

Während des Eingriffes steht dem Operateur lediglich die radiologische Kontrolle mit Bildverstärker zur Verfügung. Insbesonders im Bereich des kervikothorakalen Übergangs kann die Einsicht schwierig bis unmöglich sein.

Immer ist mit geeigneten Lagerungstechniken darauf zu achten, dass insbesondere diese Regionen bereits präoperativ ausreichend einsehbar sind. Dienlich sind beidseitig angelegte Unterarmmanschetten, mit deren Hilfe intraoperativ kurzfristig beide Schultern nach kaudal gezogen werden können. Um Armplexusschädigungen zu vermeiden, darf dieser Zug nur so kurz wie nötig, keinesfalls über die Dauer der gesamten Operation ausgeübt werden.

Die intraoperative radiologischen Repositionskontrolle von verhakten, insbesonders einseitig verhakter Luxationen kann infolge suboptimaler Bildverhältnisse schwierig sein. Hier empfiehlt es sich, den Bildverstärker beidseits in anterior-posteriorer Ansicht schräg einzuschwenken, um so optimal die Gelenksstellungen beurteilen zu können.

Bei verbliebener Restkyphose gilt es zu beurteilen, inwieweit ein zu niederer oder zu wenig keilförmiger Knochenspan interponiert wurde. Der häufigste Fehler in diesen Fällen liegt allerdings darin, die ventrale Platte nicht entsprechend der Halslordose im operierten Segment vorzubiegen.

Bei Verhakung einer von ventral fixierten Verrenkung kann, so wie auch im Rahmen der Erstversorgung, nach Metallentfernung durch eine Kombination aus externem Zug am Schädel (z.B. über eine Haloring-Extension) und einem von ventral interkorporell einseitig einge- brachten Clowards-Spreizer die dorsale Enthakung herbeigeführt werden.

\section{Redislokationen}

Frühe postoperative Redislokationen sind selten Folge von nicht fachgerechten äußeren Manipulationen oder erneuten Stürzen, sondern finden ihre Ursachen zumeist in einer Fehleinschätzung des Frakturtypus.

Besonders Augenmerk ist dabei auf vermeintlich rein kraniale Berstungsbrüche zu legen, bei welchen oft eine Teil-
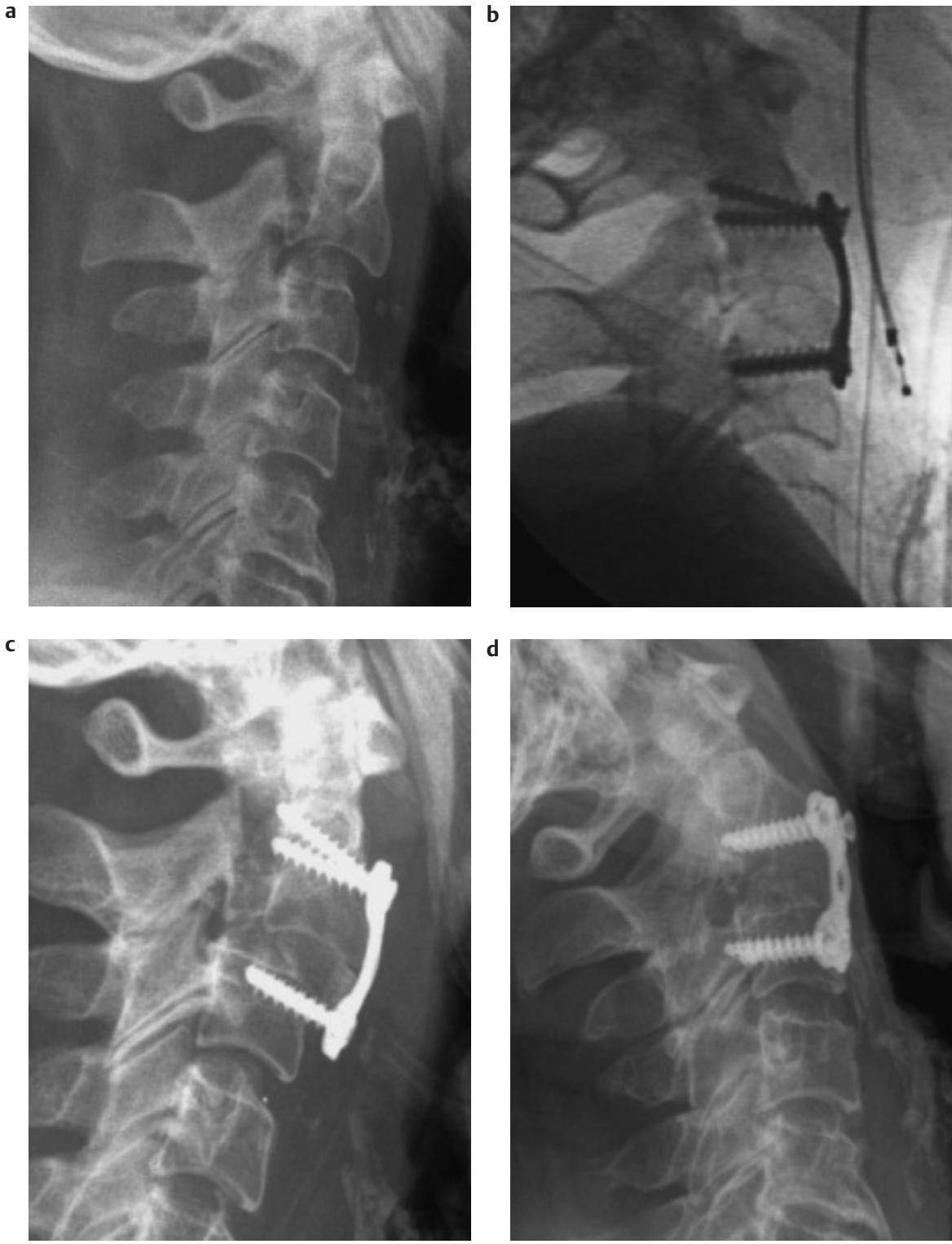

Abb. 3 S.M. weiblich 73a, traumatische Spondylolyse C2 (Typ Effendi 2) im Rahmen eines Sturzgeschehens. Nach gedeckter Reposition und interkorporeller Spanbolzung Anlage einer H-Platte. Die Verankerung der Titanschrauben erfolgten routinemäßig nicht in der Hinterwand (a und $\mathbf{b}$ ). Die radiologische Verlaufskontrolle nach sechs Tagen zeigte die Redislokation infolge des kaudalen Schraubenausrisses. Im Rahmen der Respondylodese wurden alle Schrauben hinterwandperforierend verankert ( $\mathbf{c}$ und $\mathbf{d}$ ). korporektomie und monosegmentale Instrumentation ausreicht. Wird dabei aber ein Berstungsspaltbruch übersehen, und dieser monosegmental versorgt, so können im ungünstigen Falle die Schrauben im sagittal verlaufenden Bruchspalt zu liegen kommen. Eine ImplantatdislokatiRevision ist eine klassische Korporektomie und bisegmentale Fusion und Instrumentation durchzuführen (Abb.2).

Ventrale Implantate wurden seit ihren ersten Anwendungen ständig weiterent- 
wickelt. Über lange Zeit war es zwingend a nötig, die zugehörigen Stahlschrauben nach Perforation der Wirbelkörperhinterwand dort zu verankern, um gefürchtete Implantatdislokationen zu vermeiden. Titanplasmabeschichtete Schrauben und Titanimplantate mit spezieller Oberflächenstruktur machen diese nicht ganz ungefährliche Anforderung unnötig. Die fehlende Verankerung dieser Implantate in der hinteren Kortikalis kann bei reduzierter Knochendichte zu einer geringeren Primärstabilität und damit in selten Fällen zu Frühdislokationen führen [10].

Wir beobachteten Frühdislokationen einerseits im Rahmen von ventralen Fusionen nach traumatischen Spondylolysen C2 und konsekutiver Subluxation C2/C3, andererseits bei Bechterew-Frakturen.

Aufgrund der deutlich erhöhten Hebelkräfte stellen letztere die Indikation zu langstreckigen und zumeist dorsoventralen Stabilisierungen dar. Nachdem es sich in diesen Fällen um Frakturen vergleichbar am Schaftknochen handelt, sind Knochentransplantationen selbstverständlich nicht notwendig.

Im ventralen Revisionsfall nach Subluxation infolge Implantatdislokation genügt zumeist die gedeckte Reposition und Fixation der Schrauben in den Wirbelkörperhinterwänden (Abb.3).

\section{Spätkomplikationen nach Spondylodesen an der HWS}

\section{Implantatversagen}

Unter Implantatversagen versteht man eine im postoperativen Verlauf eintretende Änderung dessen struktureller Integrität, gleich ob sich dies nun in einem Metallbruch oder beispielsweise in der Lösung einer Stab-Schrauben-Verbindung manifestiert. Die Ursachen liegen selten im Implantat selbst, sondern sind zumeist der negative Endpunkt einer Abfolge von intra- und postoperativen Fehlern.

Implantatversagen und das Versagen der biologischen Knochenheilung stehen wie überall am Skelett auch an der Wirbelsäule in direktem Zusammenhang. Kommt es zu keiner knöchernen Konsolidation an der Grenze zum Transplantat und Knochenlager, so kann eine oft schmerzhafte Restbeweglichkeit im pseudoarthrotischem Segment entstehen (Abb.4). Ein Implantatversagen, unabhängig von dessen Design und anatomi-
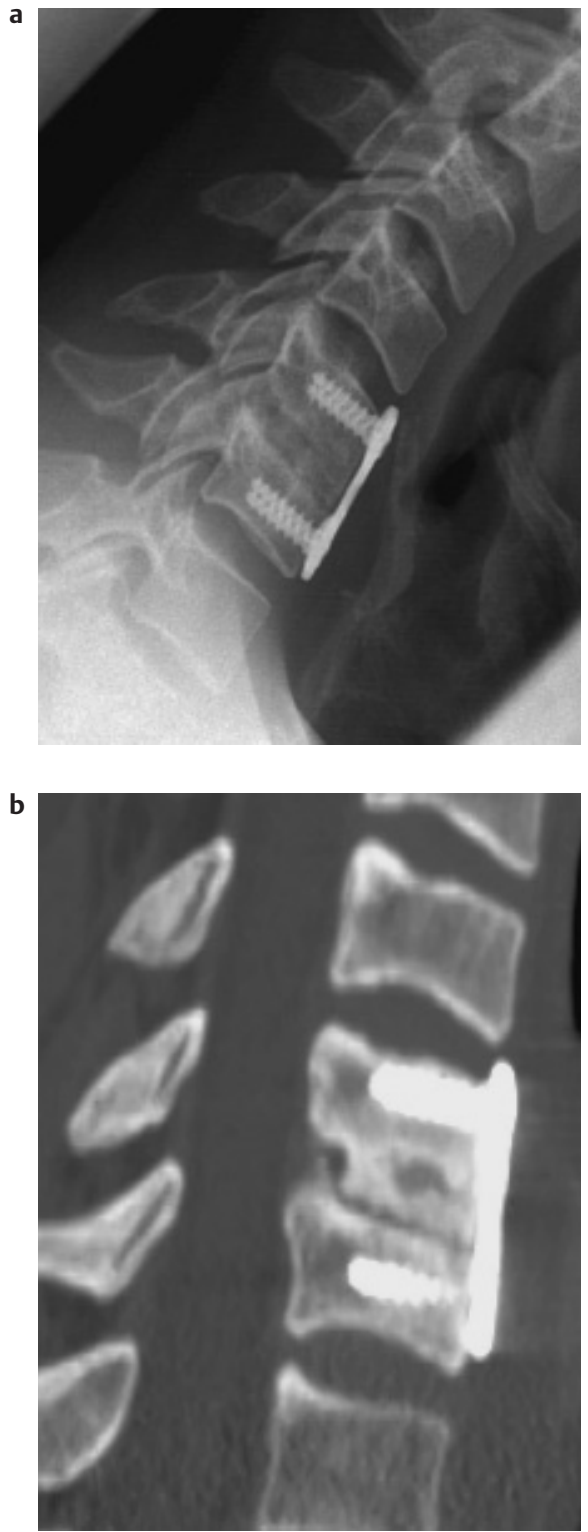

Abb. 4 P.G. 40 Jahre. Bei Zustand nach zwei dorsalen Eingriffen (Foraminotomien C5/C6 und C6/C7) wurde eine ventrale Spanbolzung und Plattenfusion C5/C6 durchgeführt. Ein Jahr nach diesem Eingriff bestehen unverändert bewegungsabhängige Beschwerden in der mittleren HWS. Die CT zeigt die Pseudoarthrose infolge unzureichender Spaneinheilung (a und $\mathbf{b}$ ).

scher Lage, wird die unweigerliche Folge sein (Abb.5).

Die Präparation des Knochenlagers an der Wirbelsäule stellt somit einen zentralen und überaus wichtigen Part des operativen Vorgehens dar. Die Verwendung einer rotierenden Fräse hat sich insbesonders in Rahmen von Uncoforaminotomien sehr bewährt und findet auch in der Zurichtung des ventralen Spanlagers gute Verwendung. Größte Vorsicht ist aber dahin- gehend geboten, keine Hitzenekrosen an den Wirbelkörperendplatten infolge zu langer und $\mathrm{zu}$ hochtouriger Anwendung zu setzen. Dies gilt insbesonders bei Dekompressionen in mehreren Segmenten, dahingehend, dass der zentrale Wirbelkörper sowohl von kaudal als auch von kranial präpariert werden muss. Einheilungsprobleme der anliegenden Knochenspäne können die Folge sein.

Auch bei dorsalem Vorgehen ist das Anfrischen des späteren Spanlagers ein elementar wichtiger Bestandteil der Operation und unbedingte Voraussetzung für eine optimale Spaneinheilung. Bei der heute seltenen alleinigen Anwendung von Drahtcerclagen an der dorsalen HWS können im postoperativen Verlauf durchaus Drahtrisse bei guter knöchernen Konsolidation beobachtet werden. In allen diesen Fällen bedarf es einer qualitativ hochwertigen CT-Diagnostik zur Beurteilung der knöchernen Konsolidation.

\section{Rekyphosierungen nach Knochenspaninterposition}

Nachsinterungen mit konsekutiver Segmentkyphose nach alleiniger interkorporeller Knochenspaninterposition im Rahmen der klassischen Cloward-Technik sind in der Literatur hinlänglich beschrieben. Bei knöchern zumindest teilfixierter Kyphose bedarf es im Revisionsfall einer ventralen Osteotomie, Interposition eines keilförmigen Knochenspanes und ventraler Plattenspondylodese. Nur in seltenen Fällen bei auch dorsal knöchern fixierten Gelenken ist das alleinig ventrale Vorgehen nicht ausreichend und eine primäres dorsales Release muss vorangehen.

\section{Rekyphosierung nach Wirbelsatz oder Teilersatzoperationen}

Wirbelersatzimplantate stellen in sich steife Konstrukte dar, welche nach Korporektomien oder Segmentresektionen implantiert werden. Diese dem biomechanischen Prinzip der Abstützung folgenden Implantate rekrutieren sich aus der Tumorchirurgie, waren früher solide Konstrukte im Sinne metallarmierter Palacos-Zylinder, Spongiosametall oder ähnliches und entwickelten sich zunehmend zu Hohlzylindern oder Quadern in Gitter- oder Körbchendesign, gefertigt aus Kunststoffen oder verschiedensten Metalllegierungen.

Ihnen allen gemeinsam ist die notwendige Steifigkeit, um eben dem Abstützungsprinzip zu genügen. 

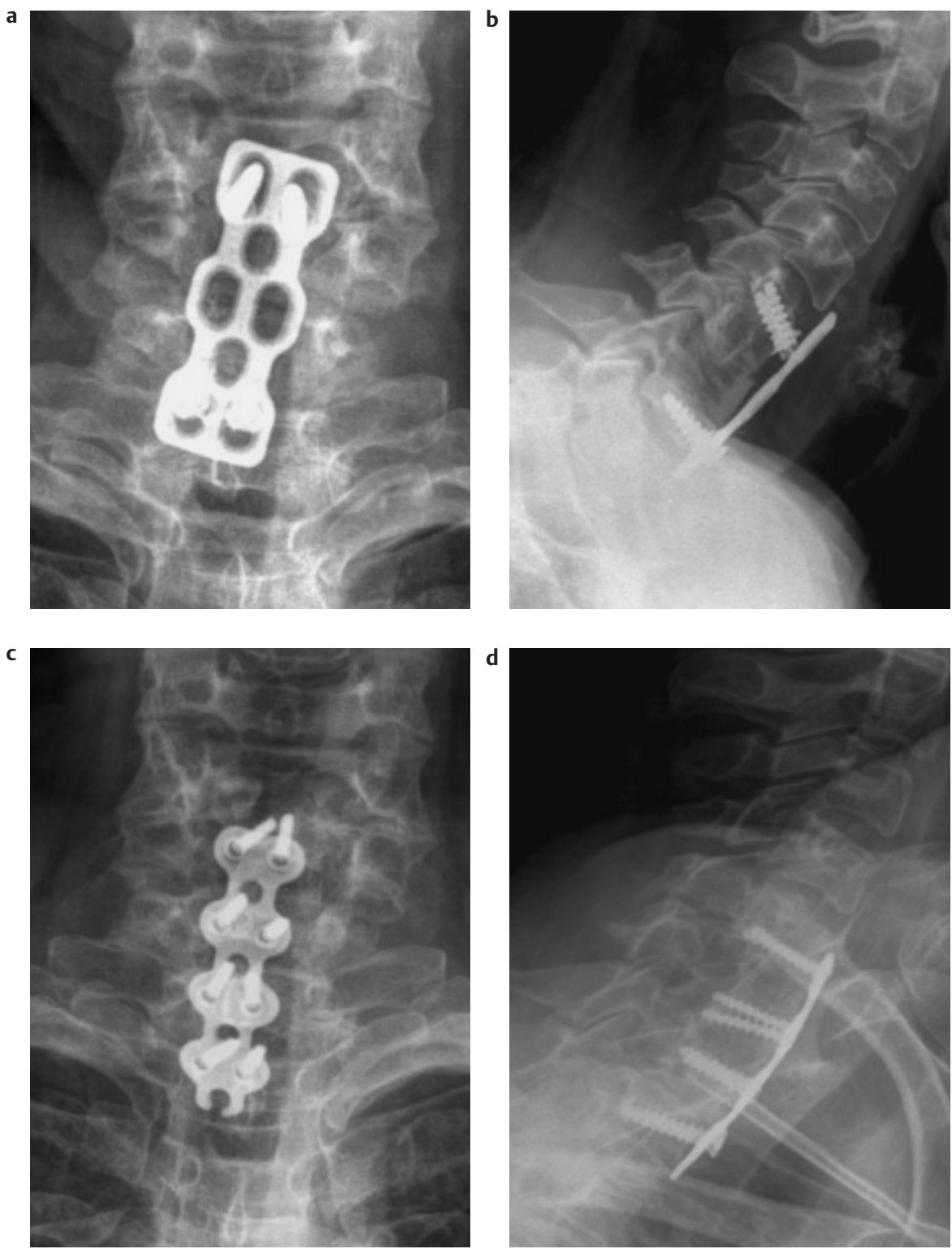

Abb. 5 K.B. 53 Jahre. Nach klassischer Cloward-OP C5/C6 vor Jahren wurde in weiterer Folge der Versuch der ventralen Respondylodese mittels ventraler Plattenanlage C5/C6 unternommen. Aufgrund unzureichender knöcherner Konsolidation kam es zum Implantatversagen, Instabilitätsbeschwerden sowie radikulären Schmerzen C6 und C7 (a und b). Nach Metallentfernung, Pseudorthrosenausräumung, Uncoforaminotomien C5/C6 und C6/C7 und interkorporellen Spanbolzungen erfolgte die ventrale Instrumentation C5 bis TH1 (c und d).

In den letzten längeren Jahren fanden diese Implantate zunehmend auch ihren Weg aus der tumorchirurgischen Anwendung in den degenerativen und auch traumatologischen Bereich im Bestreben, die sonst notwendige Knochenspanentnahme mit ihrer bekannten lokalen Morbidität zu umgehen.

Mit nun deutlich längerer Verweildauer dieser Implantate im Gegensatz zu palliativchirurgischen Anwendungen und auch oft biomechanisch anderen Anforderungen vor allem im traumatologischen Be- reich traten sehr bald die Negativseiten dieser starren, biologisch nicht adaptierbaren Implantate in den Vordergrund.

Dislokationen sowohl nach ventral oder dorsal, aber auch nach zentral im Sinne des Einbruches der Grund- oder Deckplatte, Knochenarosionen an den Implantat-Knochen-Lagergrenzen sowie Instabilitäten sind gefürchtete Komplikationen, insbesonders dann, wenn derartige Implantate im „Stand alone“ - Prinzip ohne ventrale Instrumentation eingebracht werden $[11,12,13]$.

\section{Literatur}

${ }^{1}$ Smith GW, Robinson RA. The treatment of certain cervical-spine disorders by anterior removal of the intervertebral disc and interbody fusion. J Bone Joint Surg Am 1958 Jun; 40-A(3): 607-624

${ }^{2}$ Cloward RB. The anterior approach for removal of ruptured cervical disks. J Neurosurg 1958 Nov; 15(6): 602-617

${ }^{3}$ Orozco-Delclos R, Llovet-Tapies J. Osteosintesis en las fracturas de rachis cervical. Revista Orthop Traumatol 1970; 14: 285-288

${ }^{4}$ Anderson P, Henley M, Grady M, Montesano $\mathrm{P}$, Winn $\mathrm{H}$. Posterior cervical arthrodesis with $\mathrm{AO}$ reconstruction plates and bone graft. Spine 1991 (3 Suppl): 72

${ }^{5}$ Robinson RA, Southwick WO. Surgical approaches to the cervical spine. Instr Course Lect 1960; 17: 299-330

${ }^{6}$ Aebi M, Zuber K, Marchesi D. Treatment of cervical spine injuries with anterior plating. Indications, techniques, and results. Spine 1991 (3 Suppl): 38-45

Herkowitz H. A comparison of anterior cervical fusion, cervical laminectomy, and cervical laminoplasty for the surgical management of multiple level spondylotic radiculopathy. Spine 1988; 13(7): 774-780

${ }^{8}$ Lange U, Pape HC, Bastian L, Krettek C. Management operativer Stabilisierungen von Halswirbelsaulenverletzungen bei Morbus Bechterew. Unfallchirurg 2004 (Publ. Online)

${ }^{9}$ Lehmann W, Blauth M, Briem D, Schmidt U. Biomechanical analysis of anterior cervical spine plate fixation systems with unicortical and bicortical screw purchase. Eur Spine J 2004 Feb; 13(1): 69-75

${ }^{10}$ Lehmann W, Briem D, Blauth M, Schmidt U. Biomechanical comparison of anterior cervical spine locked and unlocked plate-fixation systems. Eur Spine J 2004 (Publ. Online)

${ }^{10}$ Pitzen T, Barbier D, Tintinger F, Steudel WI, Strowitzki M. Screw fixation to the posterior cortical shell does not influence peak torque and pullout in anterior cervical plating. Eur Spine J 2002; 11(5): 494-499

${ }^{11}$ Gercek E, Arlet V, Delisle J, Marchesi D. Subsidence of stand-alone cervical cages in anterior interbody fusion: warning. Eur Spine J 2003 Oct; 12(5): 513-516

${ }^{12}$ Kanayama M, Hashimoto T, Shigenobu K, Oha F, Ishida T, Yamane S. Pitfalls of anterior cervical fusion using titanium mesh and local autograft. J Spinal Disord Tech 2003, 16(6): $513-518$

${ }^{13}$ Vavruch L, Hedlund R, Javid D, Leszniewski W, Shalabi A. A prospective randomized comparison between the cloward procedure and a carbon fiber cage in the cervical spine: a clinical and radiologic study. Spine 2002; 27(16): 1694-1701

\section{Dr. med. Peter Seykora}

Oberarzt

\section{Dr. med. Anton Kathrein}

Oberarzt

Univ. Prof. Dr. med. Michael Blauth Klinikvorstand

Universitätsklinik für Unfallchirurgie

Anichstr. 35

A-6020 Innsbruck 